\title{
Molecular Dynamics Simulation Study of the Structures and Properties of Three Polymer Binders
}

\author{
Weihong Chai ${ }^{1,}$, , Qinghua Wei ${ }^{1, b}$, Yanen Wang ${ }^{1, c}$, Mingming Yang ${ }^{1, d}$, Xinpei \\ $\mathrm{Li}^{1, \mathrm{e}}$, Shengmin $\mathrm{We}^{1, \mathrm{f}}$ \\ ${ }^{1}$ School of Mechanical Engineering, Northwestern Polytechnical University, Xi'an, 710072, China \\ a351782325@qq.com, ${ }^{b} 598820042 @ q q . c o m,{ }^{c}$ wangyanen@126.com, ${ }^{\mathrm{d}} 726857373 @ q q . c o m,{ }^{\mathrm{e}} 892$ \\ 431954@qq.com, 'weism@nwpu.edu.cn
}

Keywords: molecular dynamics simulation; polymer binder; structure; properties.

\begin{abstract}
During the technology process of 3DP fabrication bone scaffolds, the property of binder is a key factor which affects the quality of bone scaffold. In this research, three models of polymer binders were constructed and simulated by a molecular dynamics simulation method. Some properties (such as density, cohesive energy and mechanical properties) of polymer binders PVP, PAM and PVA were investigated at molecular level. The properties of three binders were also compared, and their inner relations were revealed. Therefore, this study not only forecasts the basic properties of the commonly used polymer binders, but also provides the theoretical basis for the choice of binder in technology process of 3DP fabrication bone scaffolds.
\end{abstract}

\section{Introduction}

With the development of 3DP technology, the 3DP fabrication technology based on the principle of micro-droplet has been widely used in many fields, especially in the areas of bone scaffold preparation [1-2]. But there are remain lots of challenges should be solved, such as mechanical properties, biocompatibility, and so on. In the preparation process of bone scaffold, the interaction between droplets and powder determines the microstructure and macroscopic mechanical property of bone scaffold. Therefore, meeting the requirements of artificial bone mechanical properties is a key point for 3DP fabrication bone scaffolds, and this is also the main factor that limits the technology widely used. Presently, the polymer binders are usually used to bond biological ceramic powder material in the process of 3DP fabrication bone scaffolds. Due to the mechanical property of ceramic material is much higher than the polymer binder, so the mechanical properties of binders play a decisive role for the mechanical properties of bone scaffold. Therefore, studying the properties of binders has the very vital significance for the bone scaffold preparation technology.

In this paper, three types of polymer binders Polyvinylpyrrolidone (PVP), polyacrylamide (PAM), and polyvinyl alcohol (PVA) which commonly used have been studied from the structures and properties by molecular dynamics (MD) simulation. By analyzing the equilibrium models, many basic properties about binders can be forecasted form the molecular level, this provides the theoretical guidance for the selection of polymer binders in technology of 3DP fabrication bone scaffolds.

\section{Materials Models and simulation methods}

Due to the limitations of computer calculating ability, and the purpose of this article is intended to reveal the structures and properties of PVP, PAM and PVA, therefore, the water is not taken into account while building the models. Firstly, according to the molecular structure formula of PVP, PAM and PVA (Fig.1), three molecular chains were constructed respectively. To compare with each other, we had better make the molecular weights of the binder molecular chains close to each other, then the repeat unit numbers of PVP, PAM and PVA molecular chains were 20, 31 and 50, The detail calculation process can refer to our previous research work [3-4]. Following the simulation models of 
polymer binders were built with the Amorphous module in the Materials Studio, their initial densities of the PVP, PAM and PVA were all set as $0.6 \mathrm{~g} / \mathrm{cm}^{3}$. After geometry optimization, MD simulations were performed. Here the MD simulation contains the following two steps. (1) MD simulation under NPT ensemble, the calculation time was 200 ps, or stopping calculation after the density of system achieve balance. (2) MD simulation under NVT ensemble, simulation time was set 50 ps, and the numerical analysis was carried on the trajectory frames obtained in this process.

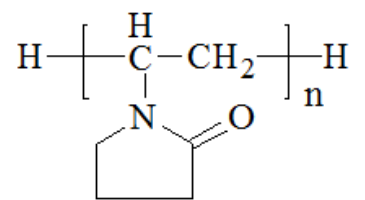

(a) PVP<smiles>C[14CH2]C[C@H](C(N)=O)C(C)C</smiles>

(b) PAM<smiles>CCC(O)CC(C)C</smiles>

(c) PVA

Fig.1 Molecular structural formula of (a) PVP, (b) PAM and (c) PVA

All the MD simulations above were performed under the force of COMPASS [5], the Anderson thermostat and barostat [6] were used to maintain the temperature and pressure for all NVT and NPT MD simulations. The Coulomb and van der Waals long-range nonbonding interactions were handled using the standard Ewald and Atom-Based summation methods [7-8], respectively. As well, nonbonding interaction was truncated at $1.25 \mathrm{~nm}$. A time step of $1 \mathrm{fs}$ was used for all MD simulation. Fig. 1 shows the equilibrium structures of three polymer binders. Table 1 lists the initial densities, the number of chains and atoms, and the final densities of all simulated polymer amorphous cells at the temperature $298 \mathrm{~K}$.

Table 1 Detailed parameters of amorphous unit cells

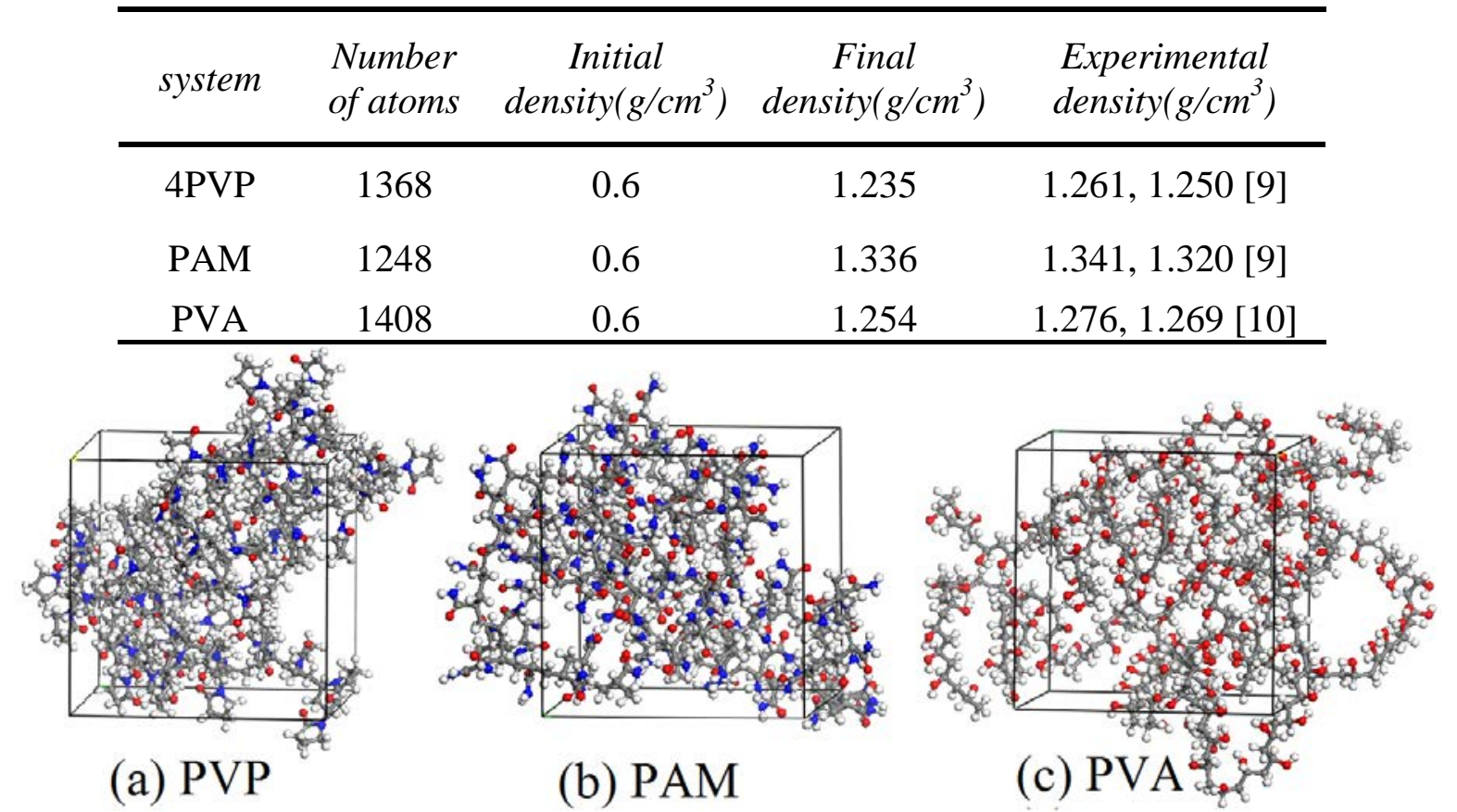

Fig.2 Equilibrium models of three binders. Each amorphous cell contains four molecular chains.

\section{Results and Discussion}

\subsection{The judgment of the system balance}

The equilibrium of simulation models is the premise condition for the materials properties analysis, and the energy, temperature, density, and radial distribution function were all can be used to judge whether the system is equilibrium. Herein, the density and energy were used to judge the equilibrium of simulation system. When the densities and energies of models were fluctuating around a constant value, the system can be thought equilibrium. Fig. 2 is the curves of the density and energy of PAM system changing with simulation time, and from it we can know the fluctuations of curves in figures 
are very slight, which indicates the system reaches equilibrium after MD simulation under NPT ensemble.
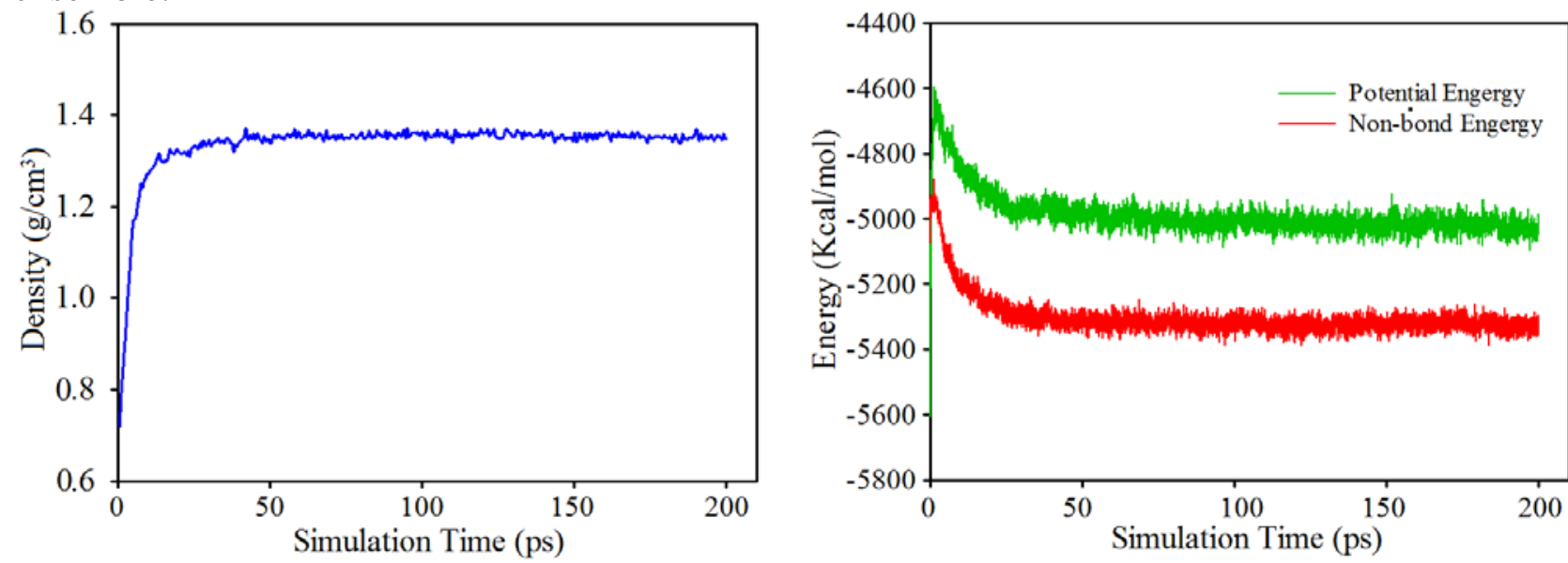

Fig.2 Plot of the density and total potential energy, nonbond energy of 2PAM binder system as a function of MD simulation time.

\subsection{Cohesive energy density}

Cohesive energy density (CED) is an improtant parameter for polymer binder, which can be used to evaluate the interaction force between molecules in system, also it can reflect the interaction among functional groups. Therefore, the properties related to material interactions are intrinsically linked to cohesive energy, such as solubility, compatibility, viscosity, and so on. This is defined as Equation 1.

$$
C E D=\frac{E_{\text {coh }}}{v}
$$

Where $E_{c o h}$ is cohesive energy, $v$ represents the mixed volume of materials, and CED is the cohesive energy density. In general, the stronger the polarity of groups in molecules, the bigger the interaction force between molecules, and the $C E D$ is also bigger.

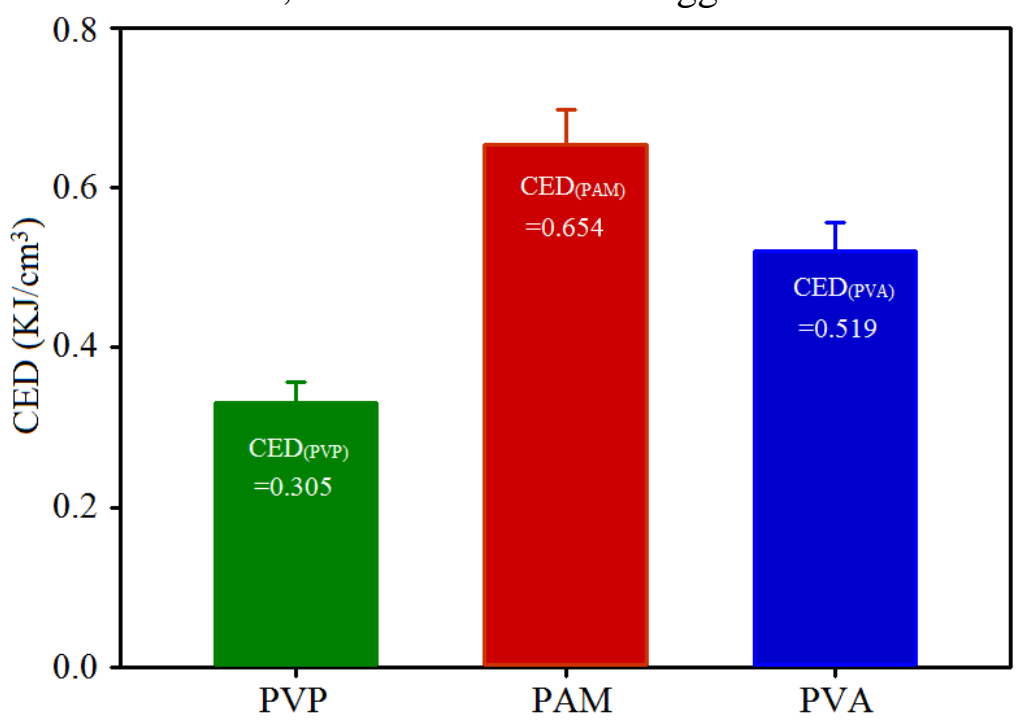

Fig.3 CED Histograms of three polymer binders

From the calculated results of $C E D$ for three polymer binders in Fig.3, we can easily see that the $C E D$ size relation of three binders has the order of PAM $>\mathrm{PVA}>\mathrm{PVP}$, that is to say the CED of PAM is the biggest in these three polymer binders, then is PVA. While the size of CED can reflect the strength of interaction between molecules in system, so the size relation of interaction between molecules in three polymer binder systems is PAM $>$ PVA $>$ PVP, this is consistent with the density of binders. 


\subsection{Mechanical properties}

In process of 3DP fabrication bone scaffolds, the mechanical property of binders directly relates the strength of bone scaffold, so the mechanical property is also one of the most important parameters for binders. This article used the static mechanics analysis method to calculate the mechanical properties of equilibrium systems (such as elastic coefficients, modulus, Poisson's ratio, et al). At the atomic level calculation, the virial equation was used to calculate the internal stress tensor $(\sigma)$ [11] of the static model.

$$
\sigma=-\frac{1}{V_{0}}\left[\sum_{i=1}^{N} m_{i}\left(V_{i}, V_{i}^{T}\right)\right]
$$

Where mi and Vi represent the quality and speed of atoms, respectively, and $V_{0}$ is volume without distortion.

The elastic coefficient matrix is obtained by solving the first derivative of the corresponding stress and strain. Static elastic properties, i.e., tensile (Young's) modulus $(E)$, bulk modulus $(K)$, and shear modulus $(G)$, and Poisson's ratio $(\gamma)$ were calculated as follows [12].

$$
E=\frac{\mu(3 \lambda+2 \mu)}{\lambda+\mu}, \quad K=\lambda+\frac{2}{3 \mu}, \quad G=\mu, \quad \gamma=\frac{\lambda}{2(\lambda+\mu)}
$$

Where $\mu$ and $\lambda$ are the Lamé coefficients. Generally, the hardness of material is closely related with $E$ and $G$, the larger the value of $E$ and $G$, the higher the rigidity and hardness of the material. The fracture strength of material is determined by $K$, a higher value of $K$ indicating a greater ability of fracture resistance. Moreover, Cauchy pressure is usually used to forecast the ductility of materials, if the value of Cauchy pressure is negative, then the material is brittle, while the positive value indicating the material is tough. The higher the Cauchy pressure value, the better the material's ductility [13-14]. Table 2 shows the elastic coefficients of binders, and Fig. 4 shows the static elastic properties which calculated from the data in Table 2.

Table.2 Elastic coefficients of binders at 298K (GPa)

\begin{tabular}{cccccccccccccc}
\hline System & $\mathrm{C}_{11}$ & $\mathrm{C}_{22}$ & $\mathrm{C}_{33}$ & $\mathrm{C}_{44}$ & $\mathrm{C}_{55}$ & $\mathrm{C}_{66}$ & $\mathrm{C}_{12}$ & $\mathrm{C}_{13}$ & $\mathrm{C}_{23}$ & $\mathrm{C}_{15}$ & $\mathrm{C}_{25}$ & $\mathrm{C}_{35}$ & $\mathrm{C}_{46}$ \\
\hline PVP & 9.53 & 8.54 & 7.35 & 1.80 & 2.91 & 2.39 & 4.50 & 3.87 & 3.16 & -1.14 & -0.65 & 0.17 & -0.20 \\
PAM & 5.77 & 6.08 & 4.56 & 2.39 & 2.76 & 2.12 & 4.27 & 3.55 & 3.58 & 0.84 & 0.32 & -0.48 & -0.19 \\
PVA & 15.99 & 14.23 & 14.95 & 2.23 & 3.64 & 4.02 & 7.89 & 7.16 & 10.25 & -0.27 & -0.62 & 0.12 & 0.13 \\
\hline
\end{tabular}

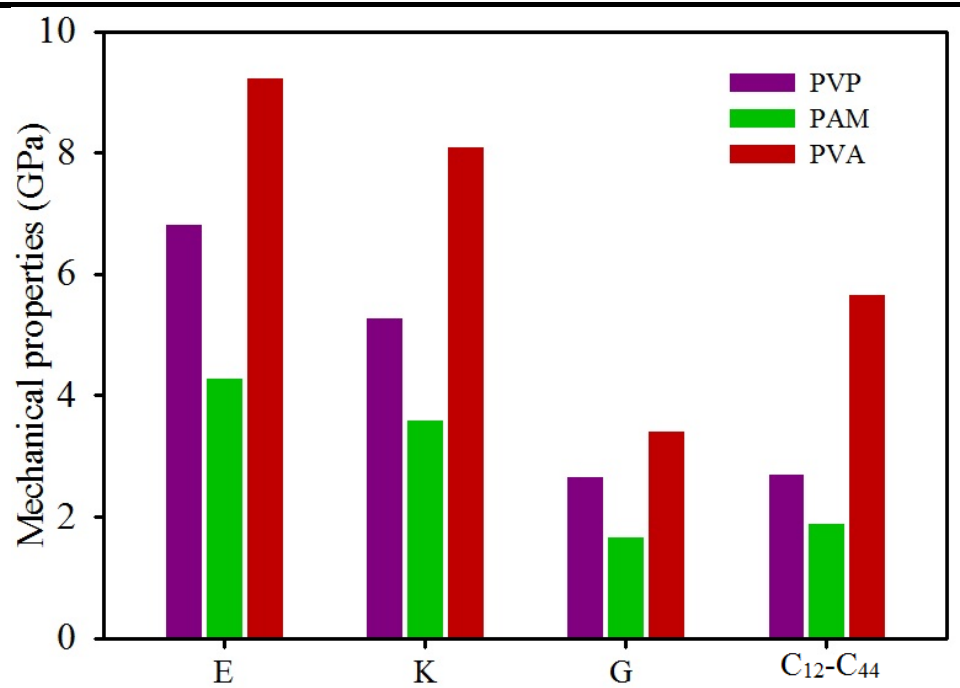

Fig. 4 Histogram of mechanical properties for three binders

From Fig.4, we can find there is a certain difference in mechanical properties of three binders, and size relationships of modulus and Cauchy pressure for three kinds of binders were consistent with each other, the order is PVA $>$ PVP $>$ PAM. Namely, compared with PVP and PAM, the mechanical properties of PVA is better, which is inconsistent with the result of cohesive energy, indicating there 
is no certain relationship between the mechanical properties and cohesive energy, density for polymer binders. Additionally, the Cauchy pressure values of three binders are all positive, which indicates that three binders above have some ductility, and the ductility of PVA is the best, PAM is the worst.

\section{Conclusion}

The models of three commonly used polymer binders PVP, PAM and PVA were constructed and simulated by a molecular dynamics simulation method, the computational analysis was conformed on the equilibrium models, and some properties (density, cohesive energy, mechanical properties) were calculated in this paper. The results show that the size relation of CED for three binders has the order as PAM $>$ PVA $>$ PVP, which is consistent with the size relation of density. Furthermore, results of static mechanic analysis indicates the mechanical strength size relation of binders is PVA $>$ PVP $>$ PAM, which is inconsistent with the results of CED and density, this shows that there is a no specific relationship between mechanical properties and CED.

\section{Acknowledgements}

This project is sponsored by the National Natural Science Foundation of China, (Grant No. 51175432), the Doctor Special Science and Technological Funding of the China Ministry of Education (Grant No. 20116102110046), the Fundamental Research Funds for the Central Universities (Grant No. 3102014JCS05007), the key Industrial Science and technology projects of Shaanxi (No.2015GY047), and the Xinjiang Uygur Autonomous Region science and technology project (Grant No. 201130112).

\section{References}

[1]Chiu C K, Ferreira J, Luo T J, et al. Direct scaffolding of biomimetic hydroxyapatite-gelatin nanocomposites using aminosilane cross-linker for bone regeneration [J]. J Mater Sci Mater Med, 2012, 23(9): 2115-2126.

[2]Yanen Wang, Xinpei Li, Qinghua Wei, Mingming Yang, et al. Study the mechanical properties of three-dimensional directly binding hydroxyapatite powder [J]. Cell Biochem Biophys, (2015)72: 289-295.

[3]Qinghua Wei, Yanen Wang, Xipei Li, Mingming Yang, et al. Study the bonding mechanism of binders on hydroxyapatite surface and mechanical properties for 3DP fabrication bone scaffolds [J]. J. Mech. Behav. Biomed. Mater., 57 (2016) 190-200.

[4]Qinghua Wei, Yanen Wang, Weihong Chai, Tao Wang, et al. Effects of composition ratio on the properties of poly(vinyl alcohol)/poly (acrylic acid) blend membrane: A molecular dynamics simulation study [J]. Materials and Design, 89 (2016) 848-855.

[5]Sun H. COMPASS: An ab initio force-field optimied for condensed-phase application-Overview with details on alkane and benzene compounds [J]. The Journal of Physical Chemistry B, 1998, 102: 7338-7364.

[6]Andersen H C. Molecular dynamics simulations at constant pressure and /or temperature [J]. Journal of Chemical Physics, 1980, $72: 2374-2383$.

[7]Tosi M P. Cohesion of ionic solids in the Born model [J]. Solid State Physics, 1964, 16: 1-120.

[8]Ewald P P. Evaluation of optical and electrostatic lattice potentials [J]. Annals of Physic, 1921, 64: 253-287.

[9]Schildknecht, C. Vinyl and Related Polymers [M]. Wiley and Sons, New York, 1952. 
[10] Shijin Dong, Juntao Yan, Nan Xu, Jia Xu, et al. Molecular dynamics simulation on surface modification of carbon black with polyvinyl alcohol [J]. Surface Science, 605 (2011) 868-874.

[11] Swenson, R.J. Comments on virial theorems for bounded systems [J]. Am. J. Phys, 51 (1983) 940-942.

[12] Watt J. P., G. F. Davies, R. J. O’Connell, The elastic properties of composite materials[J]. Rev. Geophys .Space Phys, 1976, 14: 541-563.

[13] Qinghua Wei, Yingfeng Zhang, Yanen Wang, Weihong Chai, et al. Measurement and modeling of the effect of composition ratios on the properties of poly(vinyl alcohol)/poly(vinyl pyrrolidone) membranes [J]. Materials and Design, 103 (2016) 249-258.

[14] Weiner, J. H. Statistical Mechanics of Elasticity[M]. Dover Publications Inc, New York, 2002. 